\title{
Investigating Transmission Power Control Strategy for Underwater Wireless Sensor Networks
}

\author{
Syed Agha Hassnain Mohsan ${ }^{1}$ \\ Department of Electrical Engineering \\ COMSATS University Islamabad (CUI) \\ Islamabad, Pakistan
}

\author{
Hussain Amjad ${ }^{2}$ \\ Department of Marine Science and Information Technology \\ Ocean College, Zhejiang University \\ Zhoushan, China
}

\author{
Alireza Mazinani ${ }^{3}$ \\ School of Electronic and Information Engineering \\ Beihang University \\ Beijing, China
}

\author{
Sahibzada Adil Shahzad ${ }^{4}$ \\ Department of Computer Science \\ National Chengchi University \\ Taipei, Taiwan
}

\author{
Mushtaq Ali Khan ${ }^{5}$ \\ Marine Ecology Lab, Department of Marine Science \\ Ocean College, Zhejiang University \\ Zhoushan, China
}

\author{
Asad Islam ${ }^{6}$ \\ School of Energy and Power Engineering \\ Beihang University \\ Beijing, China
}

\author{
Arfan Mahmood ${ }^{7}$ \\ Complex Networks and Control Lab \\ Shanghai Jiao Tong University \\ Shanghai, China
}

\author{
Ahmad Soban ${ }^{8}$ \\ Balochistan University of Information Technology \\ Engineering and Management Sciences (BUITEMS \\ Quetta, Balochistan
}

\begin{abstract}
Underwater wireless sensor network (UWSN) has proven its high stature in both civil and military operations including underwater life monitoring, communication and invasion detection. However, UWSNs are vulnerable to a wide class of power consumption issues. Underwater sensor nodes consume power provided by integrated limited batteries. It is a challenging issue to replace these batteries under harsh aquatic conditions. Thus, in an energy-constrained underwater system it is pivotal to seek strategies for improving the life expectancy of the sensors. In this paper, we propose transmission power control mechanism for underwater wireless sensor networks (UWSNs). We experimentally investigate the impact of transmission power and propose a control mechanism to enhance the performance of underwater wireless sensor network. In this proposed mechanism, source nodes will adjust its transmission power according to the location of destination node. This paper aims to provide a mechanism which is incorporated in SEEC. This study also outlines the mathematical modeling for proposed idea. Moreover, we have compared results of our scheme with previous implemented schemes.
\end{abstract}

Keywords-Component; underwater wireless sensor networks; transmission power; sensor; power consumption

\section{INTRODUCTION}

We have witnessed the penetration of wireless sensor networks (WSNs) into every avenue of our lives, ranging from environmental monitoring, biological medicine, national defense, underwater vehicles to marine observations [1]. A considerable research has been carried out on terrestrial sensor networks in different aspects but currently research community is attracted towards a new era of research in Underwater Wireless Sensor Networks (UWSNs). Human beings are unable to work at high pressure ocean environment for a long time. On the other hand, Terrestrial Wireless Sensor Networks (TWSNs) protocols are not feasible for UWSNs because signals transmission is elusive due to higher attenuation of aquatic environment.

Generally, UWSNs differ from TWSNs in multiple stringent design constraints such as sensors cost, dense deployment of these sensors, communication method and maximum power required for communication. The major challenges in deployments of UWSN are limited battery sources, memory and computational power. It is clear that the problem of limited battery sources is important and it is a big challenging issue for research fraternity to achieve long operation time without effecting performance of UWSN. In this new paradigm, UWSN should fulfill, despite transmission power and energy limitations, the communication requirements of several advanced technologies and manufacturing methods. Though, research community has provided several techniques to cope with these challenges due to variable characteristics of ocean but still gap lies for research.

This technology has already achieved a great stature among researchers. A growing research inclination has been noticed by industry as well as academia in UWSNs [2-3] with the 
progress in underwater technologies. A key challenging issue for UWSN lifetime is the maintenance as it's difficult to replace those batteries providing power to sensor nodes. Recently, researchers are working in underwater wireless power transfer to tackle these challenges. In addition, TWSNs routing protocols cannot be implemented to UWSNs because of limited bandwidth, long propagation delay, energy constraints, high mobility and non-uniform 3D topology. Usually underwater communication is different from air due to varying conductivity, permittivity and permeability [4-5] parameters. Some researchers proved that mobile sink is an effective way to enhance throughput. In order to collect data, these mobile sinks can connect to individual sensor node or full cluster. In research study discussed in [6], authors utilized two mobile sinks to gather data from sparse regions. A SEEC protocol is proposed in [7] and we have implemented our idea on it. It is noticed when both sinks are located at adjacent positions then throughput is affected due to same sensor nodes.

Power consumption plays a vital role in efficient performance of UWSN. One major issue in power constraint of UWSN as it is unrealistic to recharge or change batteries during sensor's life span. It is not feasible to recharge these batteries frequently. Maximum power consumption occurs while data communication [8]. Power adaptation on the basis of environment conditions is a good approach towards efficient power consumption. Two major factors, wireless link quality and distance, affect the power consumption in any wireless sensor network. The link quality depends on climate conditions and physical barriers. Transmission power is controlled to obtain a good link providing successful data delivery. On the other hand, it is preferred to adopt minimum transmission power which can extend the WSN lifetime. Reliability insurance and power optimization appears as an important concern while dealing with WSN [9]. In this context, we have provided transmission power control mechanism. Reducing transmission power can put counterproductive effects to network performance. We are interested to understand better spectrum efficiency of UWSN through efficient control of transmitted power. It has the potential to minimize power consumption in UWSN. However, it still faces issues in real world deployments. Such mechanism must be robust against dynamic and complex wireless properties [10]. Through MATLAB simulation, we analyze the performance of UWSN. The obtained results of throughput, residual energy and network stability are better through power control mechanism.

In a systematic review [11], modulation techniques and channel coding is briefly discussed for discrete underwater sensor nodes. In [12], distributed algorithms are proposed to lower communication energy consumption in WSN through reducing transmission power of sensors. The appropriate selection of transmission range is discussed in [13] which greatly influences network connectivity and energy efficiency. Power consumption control problem is also briefly addressed in [14] where authors have elaborated dynamic power management.

Considering above discussion, we were inspired and implemented a power control mechanism in SEEC. The overriding objective of this study is to seek an intelligent use of transmitted power. We have implemented a previous routing scheme SEEC with incorporating a power control mechanism. We have compared our obtained results with SEEC, DBR, EEDBR and TESM. We have organized our paper as: in Section 2, we have made relevant research discussion. Section 3 will focus on transmission power control strategy. While results discussion has been carried out in Section 4. In last sections, we have presented performance tradeoff, conclusion and future work.

\section{RELEVANT RESEARCH WORK}

Since many years' researchers have been focusing to design several UWSNs protocols for qualitative and effective research analysis. Research community is working with the aim of paving a way for new era of underwater actuation and monitoring applications. UWSNs will help us to know our ocean with its envisioned landscape of applications. Different topologies, power control mechanisms, power management algorithms and adaptive techniques have been implemented in Wireless Sensor Networks (WSNs). Chincoli et al. [15] have presented a self-learning power control system for WSN. A topology control technique for efficient energy consumption is proposed in [16] based on power management and power control. A dynamic power management technique to enhance energy efficiency is discussed in [17]. Researchers have suggested various routing scheme to achieve better results as authors in [18] highlighted capabilities and limitations on the basis of a survey on UWSN with data aggregation. Feng Pan et al. [19] presented an energy-balanced algorithm based on energy level partition and depth threshold. In this protocol, mobile sinks can collect data at sparse region and they implied clustering on dense regions. Wan et al. [20] suggested an energy efficient scheme based on multilevel cluster selection criteria which aim to choose cluster head according to high residual energy and less radius from sink. An energy efficient routing protocol based on clustering is simulated in [21] for UWSNs. Authors proposed that clustering technique is useful to reduce energy resources consumption for a sensor network. Ma Ming et al. [22] have used a hybrid approach to maximize network lifetime and minimize the energy holes. Researchers have proposed a routing protocol AEDG which involves effective data collection through AUV [23]. In this research study, authors maximized the network lifetime in AEDG as AUV is sent to gather data from gateway nodes. The usage of multiple mobile sinks to enhance network lifetime is discussed in [24]. Authors validated that a better network lifetime can be achieved through assigning a predefined path to mobile sinks for movement.

\section{TRANSMisSiON POWER CONTROL STRATEGY}

An efficient use of power consumption in UWSNs appears as maximum throughput and enhanced lifetime. In this study we have considered routing scheme SEEC and incorporated with transmission power control mechanism. We achieved good results which will be discussed in next sections.

\section{A. Network Model}

We consider a homogenous network model in this work. In addition, location information of all sensor nodes is given to each sensor node in this network. In our proposed idea, each sensor node forms four concentric circles around it. These 
concentric circles represent different power levels. As source node maintains the location information of destination node so it will adjust the transmission power which will be in either of these concentric circles. We have discussed the concentric circles formation process in mathematical modeling section. Network model design of our proposed mechanism is shown in Fig. 1.

\section{B. Mathematicl Modeling}

In UWSN, approximately 0:68 $\mathrm{dBm}$ power is required for sending packets up to distance of one meter. We consider $\boldsymbol{R}_{\boldsymbol{s}}$ as the range of sensor node. The maximum transmission power for each sensor is calculated as:

$$
\text { Trans }_{p w r}=I * R_{s n}
$$

Here we take $\boldsymbol{I}=0: 68$, as we have divided the total transmission power into four different levels so we can calculate the radius of each concentric circle around the sensor node from following equation:

$\mathrm{R}_{\text {dist }}=\mathrm{i} *\left(\mathrm{R}_{\mathrm{sn}} / 4\right) \quad 1 \leq \mathrm{i} \leq 4$

We can calculate transmission power for calculated four radii as follow:

$$
\text { Trans }_{\text {pwri }}=I * R_{\text {dist }}
$$

If we consider the transmission range of the sensor node $\boldsymbol{R}_{\text {sn }}$ as 50 meters then Table I shows the calculated radii and power magnitude at each radius.

We have incorporated the proposed transmission power control mechanism in SEEC and MATLAB simulation results will be discussed in next section.

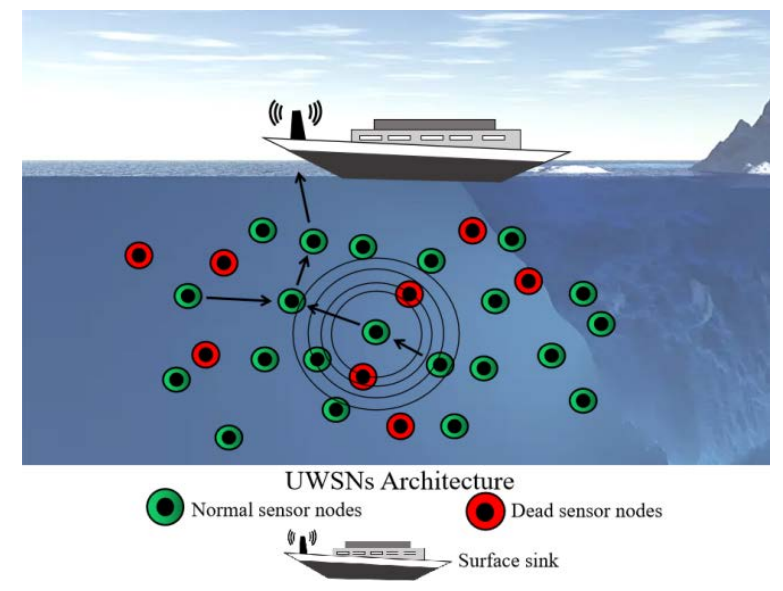

Fig. 1. Network Model.

TABLE I. RESEARCH KEYWORDS

\begin{tabular}{|l|l|}
\hline $\boldsymbol{R}_{\text {dist }}$ (Meter) & Power $\mathbf{( d B m )}$ \\
\hline 12.5 & 8.5 \\
\hline 25 & 17 \\
\hline 37.5 & 25.5 \\
\hline 50 & 34 \\
\hline
\end{tabular}

\section{Simulation RESULTS}

\section{A. Network Lifetime}

We have noticed an improvement in SEEC's network lifetime after incorporating our proposed idea of controlled transmission power mechanism. In SEEC, network was dead early as sensor nodes were sending packets with a predefined transmission power value.

While in controlled transmission power mechanism, source node adapts the transmission power on the basis of Euclidean distance from the destination node. Whenever any packet is transmitted, every time consumption power will vary. We can see a clear increase in network lifetime. In Fig. 2, nodes in SEEC were dead early while all nodes were alive till 1100 rounds after incorporating controlled mechanism.

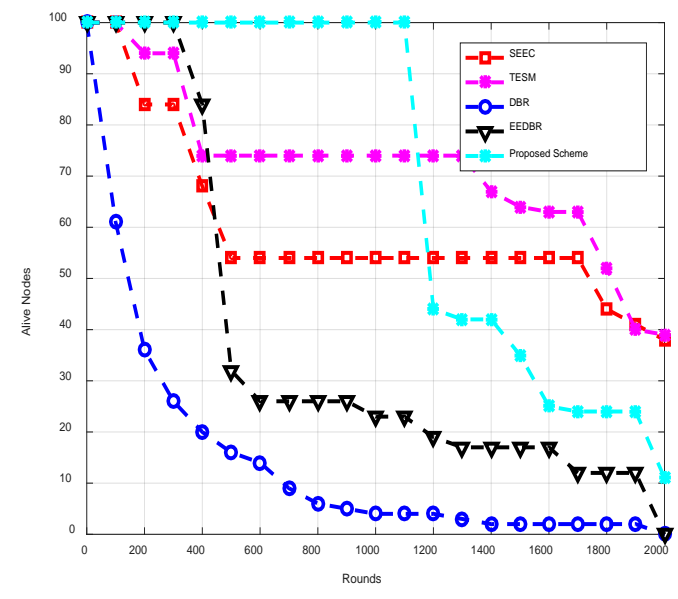

Fig. 2. Network Lifetime.

\section{B. Stability Period}

We cannot obtain same number of sparse and dense region each time through randomly deploying sensor nodes. Network stability is compromised whenever dense regions are maximums and sensor nodes communicate with same transmission power within these dense regions. On the other hand, network stability period is improved if we adapt controlled transmission power mechanism as shown in Fig. 3. In controlled mechanism, besides controlled transmission power, stability period is better than SEEC, TESM [25], DBR and EEDBR through clustering in dense regions and mobile sinks movements in sparse regions.

\section{Network Residual Energy}

The transmission power factor was constant in energy consumption equation of SEEC. While sending a packet, same energy was being consumed without considering distance between two communicating nodes. In controlled transmission power mechanism, transmitted power adjustment is made on the basis of distance between source and destination node. Low transmission power in needed in case of less distance. Low requirement of transmission power results into low energy tax providing higher network residual energy. In our proposed work, energy consumption varies its return value after transmitting the packet which also gives higher network 
residual energy. Fig. 4 shows the network residual energy for proposed scheme along with SEEC, DBR, EEDBR and TESM.

\section{Network Throughput}

In Fig. 5, the received packets per round at any sink are more than SEEC, DBR, EEDBR and TESM. We can notice an increased throughput in our proposed work with controlled transmission power mechanism. In SEEC, less packets are received as mobile sinks movement is according to sparse region and in TESM it is based on selection criteria opted for forwarding nodes.

Fig. 6 shows that the total received packets at sink are higher with controlled mechanism in SEEC. It is because of increased network lifetime by using transmission power control mechanism. EEDBR receives packets at the cost of high residual energy. In SEEC, total received packets are lower than our proposed scheme. It is because in SEEC, network is dead early and predefined transmission power also remains the same. It can be seen in Fig. 5 that received packets per round are better in our proposed controlled mechanism than SEEC, TESM, DBR and EEDBR.

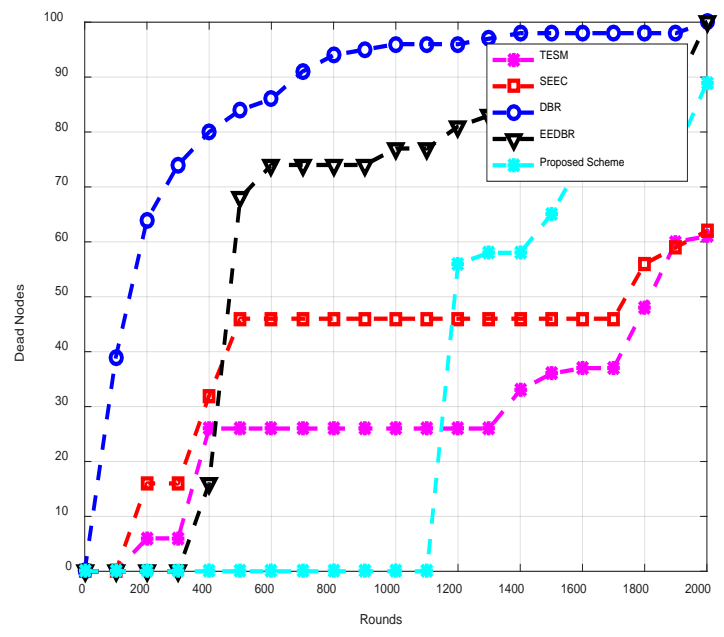

Fig. 3. Network Stability Period.

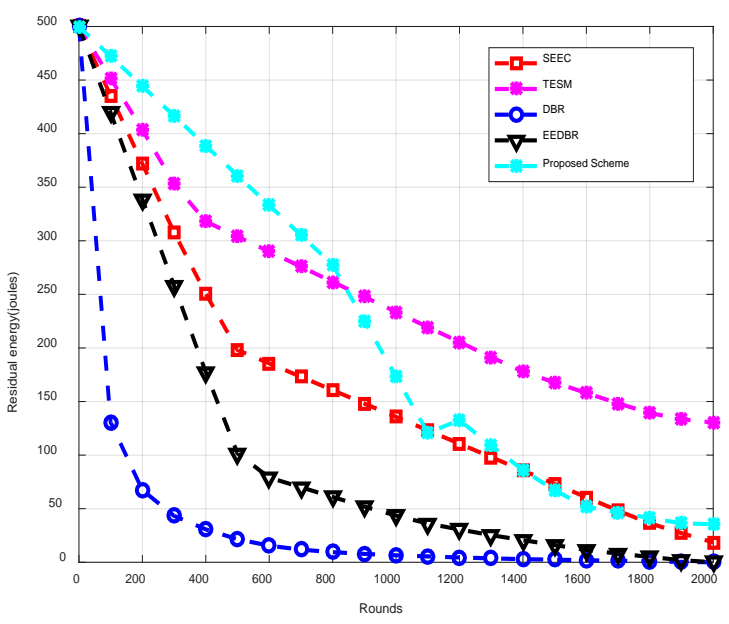

Fig. 4. Network Residual Energy.

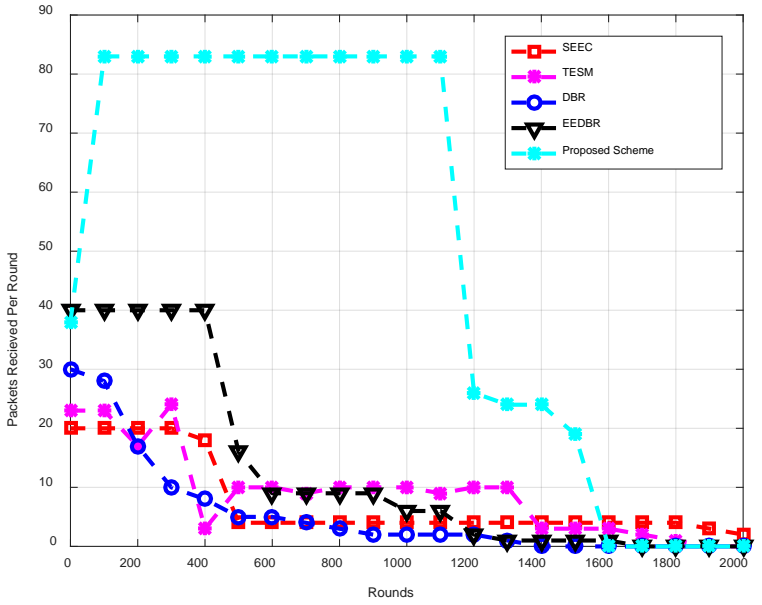

Fig. 5. Packets Received Per round.

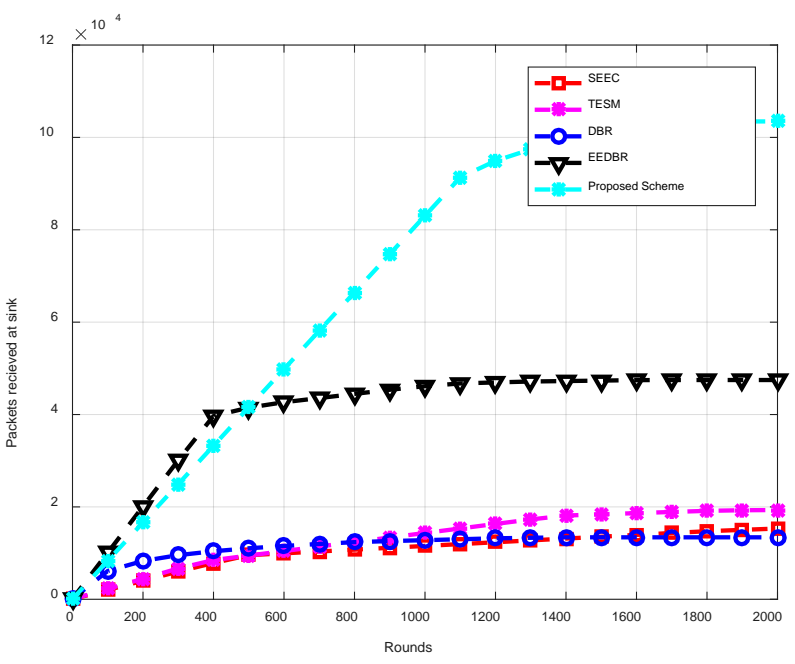

Fig. 6. Packets Received at Sink.

\section{PERformance TradeofF}

We have achieved better network lifetime, throughput and network residual energy. We can clearly see that more nodes are alive while dead nodes are less. Thus, we conclude a considerable progress of our work in aspects of stability period, residual energy and throughput by incorporating controlled transmission power mechanism.

\section{CONCLUSION AND FUTURE WORK}

Results presented in this work demonstrate that optimum and efficient use of transmission power in mobile sinks is crucially important in enhancing network lifetime and throughput. Our work has led us to conclude that a proper control mechanism for power transmission is pivotal. In addition, the results of any routing scheme can be improved if we monitor the proper use of resources in the protocol. In this study, we compared results of power control mechanism incorporated in SEEC with previous implemented schemes TESM, DBR and EEDBR. To sum up our work, the proposed idea is better in throughput, network lifetime and residual energy. Taking complex underwater environment and economic concerns into consideration, we conclude that 
UWSN should be capable to adjust itself according to environment conditions. Thus, we can achieve this through proper protocol, power control and topology design which is self-adaptive according to the changing environment conditions. However, these design characteristics should take energy efficiency into account which is crucially essential for normal operation of UWSN. In future, we are aiming to consider sparsity control in order to improve network lifetime. Holding time phenomenon towards reducing packet drop ratio is deferred to future work.

\section{REFERENCES}

[1] Luan, Zhifu. "Calculation and Simulation of Transmission Reliability in Wireless Sensor Network Based on Network Coding." International Journal of Online and Biomedical Engineering (iJOE) 13.12 (2017): 150-161.

[2] Li, N.; Cürüklü, B.; Bastos, J.; Sucasas, V.; Fernandez, J.A.S.; Rodriguez, J. A Probabilistic and Highly Efficient Topology Control Algorithm for Underwater Cooperating AUV Networks. Sensors 2017, 17, 1022.

[3] Li, N.; Martínez, J.F.; Meneses Chaus, J.M.; Eckert, M. A Survey on Underwater Acoustic Sensor Network Routing Protocols. Sensors 2016, 16, 414.

[4] Hassnain, S. A., M. J. Mughal, and Q. A. Naqvi. "Layered Chiral Spheres with Zero Backscattering." 2019 Photonics \& Electromagnetics Research Symposium-Fall (PIERS-Fall). IEEE, 2019.

[5] Hassnain, S. A., M. J. Mughal, and Q. A. Naqvi. "Analysis of Effective Medium Parameters on Polarizability of Homogeneous Chiral Sphere." 2019 Photonics \& Electromagnetics Research Symposium-Fall (PIERS-Fall). IEEE, 2019.

[6] Coutinho, Rodolfo WL, et al. "Underwater wireless sensor networks: A new challenge for topology control-based systems." ACM Computing Surveys (CSUR) 51.1 (2018): 1-36.

[7] Sher, A.; Javaid, N.; Azam, I.; Ahmad, H.; Abdul, W.; Ghouzali, S.; Niaz, I.A.; Khan, F.A. Monitoring square and circular fields with sensors using energy-efficient cluster-based routing for underwater wireless sensor networks. Int. J. Distrib. Sens. Netw. 2017, 13, 1550147717717189.

[8] Khemapech, Ittipong, Alan Miller, and Ishbel Duncan. "A survey of transmission power control in wireless sensor networks." Proc. PGNet (2007): 15-20.

[9] Lavratti, Felipe, et al. "A transmission power self-optimization technique for wireless sensor networks." ISRN Communications and Networking 2012 (2012).

[10] Fu, Yong, et al. "Practical control of transmission power for wireless sensor networks." 2012 20th IEEE International Conference on Network Protocols (ICNP). IEEE, 2012.
[11] Mehedi, Syed Agha Hassnain Mohsan Md, et al. "A Systematic Review on Practical Considerations, Recent Advances and Research Challenges in Underwater Optical Wireless Communication." IJACSA 11-7 (2020).

[12] Chen, Xiao, and Neil C. Rowe. "Saving energy by adjusting transmission power in wireless sensor networks." 2011 IEEE Global Telecommunications Conference-GLOBECOM 2011. IEEE, 2011.

[13] Gao, Mingsheng, Chuan Heng Foh, and Jianfei Cai. "On the selection of transmission range in underwater acoustic sensor networks." Sensors 12.4 (2012): 4715-4729.

[14] Mokrenko, Olesia, et al. "Dynamic power management in a wireless sensor network using predictive control." IECON 2014-40th Annual Conference of the IEEE Industrial Electronics Society. IEEE, 2014.

[15] Chincoli, Michele, and Antonio Liotta. "Self-learning power control in wireless sensor networks." Sensors 18.2 (2018): 375.

[16] Nickray, Mohsen, Ali Afzali-Kusha, and Riku Jäntti. "Simultaneous power control and power management algorithm with sector-shaped topology for wireless sensor networks." EURASIP Journal on Wireless Communications and Networking 2015.1 (2015): 118.

[17] Barceló, Marc, et al. "Joint routing, channel allocation and power control for real -Tifansactrehess

Emerging Telecommunications Technologies 26.5 (2015): 945-956.

[18] Goyal, Nitin, Mayank Dave, and Anil K. Verma. "Data aggregation in underwater wireless sensor network: Recent approaches and issues." Journal of King Saud University-Computer and Information Sciences 31.3 (2019): 275-286.

[19] Feng, Pan, et al. "Improved energy-balanced algorithm for underwater wireless sensor network based on depth threshold and energy level partition." EURASIP Journal on Wireless Communications and Networking 2019.1 (2019): 1-15.

[20] Wan, Z.; Liu, S.; Ni, W.; Xu, Z. An energy-efficient multi-level adaptive clustering routing algorithm for underwater wireless sensor networks. Clust. Comput. 2018, 1-10.

[21] Huang, Xiangdang, Shijie Sun, and Qiuling Yang. "Data Uploading Strategy for Underwater Wireless Sensor Networks." Sensors 19.23 (2019): 5265.

[22] Ma, Ming, and Yuanyuan Yang. "SenCar: An energy-efficient data gathering mechanism for large-scale multihop sensor networks." IEEE Transactions on Parallel and Distributed Systems 18.10 (2007): 14761488.

[23] Khan, Jawaad Ullah, and Ho-Shin Cho. "A distributed data-gathering protocol using AUV in underwater sensor networks." Sensors 15.8 (2015): 19331-19350.

[24] Chen, Y.S.; Lin, Y.W.Mobicast routing protocol for underwater sensor networks. IEEE Sens. J. 2013, 13, 737-749.

[25] Yahya, Aqeb, et al. "Towards efficient sink mobility in underwater wireless sensor networks." Energies 11.6 (2018): 1471. 